\title{
Palpable Breast Lesions - Cytomorphological Analysis and Scoring System with Histopatholgical Correlation
}

\author{
Dr.Sheeba.D ${ }^{1}$, Dr.Chitrakala Sugumar ${ }^{2}$ \\ ${ }^{I}$ Associate Professor, Dept Of Pathology, Govt Villupuram Medical College, Villupuram District,Tamilnadu, \\ India \\ ${ }^{2}$ Associate Professor, Dept Of Pathology, Thiruvannamalai Medical College ,Thiruvannamalai District, \\ Tamilnadu, India
}

\begin{abstract}
The study aims to correlate the Cytomorphological Diagnosis and the Modified Masood's scoring system with Histopathological Diagnosis in palpable Breast Lesions. Age distribution of cases under different categories of diagnoses were also studied. Records of the cytopathology and histopathology laboratory of Kilpauk Medical College, Chennai were reviewed over a period of 2 years. One Hundred FNAC cases were collected and the smears were stained with Hematoxylin \& Eosin and the histological confirmation were studied. Cytohistological correlation was $88 \%$ while correlation of the Modified Masood score with Histology was $84 \%$. The correlation was statistically significant with a correlation coefficient of 0.832 for CytologyHistology and 0.821 for Modified Masood-Histology. Both the correlation coefficients were significant at the 0.01 level (1-tailed). In conclusion, Modified Masood's scoring system can be done to categorize the breast lesions into the four categories which correlate highly with FNAC and histopathology.
\end{abstract}

Keywords: FNAC, Breast, Masood Score, histopathology, Correlation

\section{Introduction}

Fine Needle Aspiration Cytology is successful in identification of benign and malignant breast lesions, but its role in proliferative breast lesions is poorly defined. To expand the role of FNAC in diagnosis of proliferative breast lesions, the analysis of cytomorphological features of proliferative breast lesions in conjunction with modified Masood`s cytological scoring system and correlation with histopathology was done. This study was undertaken to categorise the breast lesions into four categories depending on nuclear dissociation, myoepithelial cells, pleomorphism of cells, anisonucleosis, nuclear chromatin and nucleoli in palpable Breast lesions. The study aims to correlate the Cytomorphological Diagnosis and the Modified Masood's scoring system with Histopathological Diagnosis . Age distribution of cases under different categories of diagnoses were also studied

\section{Materials And Methods}

The records of the cytopathology and histopathology laboratory of Kilpauk Medical College Chennai were reviewed over a period of 2 years. One Hundred FNAC cases were collected and the smears were stained with Hematoxylin \& Eosin and their histopathological confirmation done .

\subsection{Inclusion Criteria :}

All palpable breast lumps from Female patients with an adequate smear showing 5 to 6 ductal epithelial cells were included in the study. All aspirates were performed as an outpatient procedure without imaging or mammographic guidance using a $23 \mathrm{G}$ Needle and a $5 \mathrm{ml}$ syringe. The smears were fixed in Absolute alcohol for 20 minutes and then stained by Hematoxylin \& Eosin method. Aspirates were evaluated according to the Modified Masood Scoring System.

Modified Masood cytological scoring system' in which a value of 1 to 4 was given for each of the following:

\subsection{Cytological Features :}

2.2.1 Cellular Arrangement

2.2.2 Presence of Myoepithelial cells

2.2.3 Anisonucleosis

2.2.4 Cellular Pleomorphism

2.2.5 Nucleoli and

2.2.6 Chromatin Clumping

A score derived from the sum of these values was used to classify each FNA sample. 


\subsection{Scoring System}

\subsubsection{Non Proliferative Breast Disease - Score of 6 to 10 \\ 2.3.2 Proliferative Breast Disease without Atypia - Score of 11 to 14 \\ 2.3.3 Proliferative Breast Disease with Atypia - Score of 15 to 18 \\ 2.3.4 Carcinoma-Score of 19 to 24}

Cytomorphological Diagnosis and Histopathological Diagnosis were also categorised to four groups :

1. Non Proliferative Breast Disease

2. Proliferative Breast Disease without Atypia

3. Proliferative Breast Disease with Atypia

4. Carcinoma

Correlation was calculated using Spearman's rho and Kendall's tau coefficients. SPSS Statistics version 17 was used for the statistical Analysis. The Tables of data were constructed in Microsoft Office Excel 2007.

\section{Results}

The cases included in the study were all females with an Age Range of $15-65$ years. The study included 100 palpable breast lesions. Results were tabulated and analysed. The scoring system and cytomorphology correlated with histological diagnosis for 77 cases. In 12 cases, the cytomorphological diagnosis correlated well with histopathological diagnosis. In 11 cases, the modified Masood scoring system correlated well with the histological Diagnosis. In one case, the cytomorphological diagnosis categorised the case under Proliferative Breast Disease without Atypia, while the Scoring system brought it under Proliferative breast Disease with Atypia and the Histopathological Diagnosis was Carcinoma. In 2 cases, the Cytomorphological Diagnosis categorised the cases under Proliferative Breast Disease without Atypia, while the Scoring system correlated with the Histopathological Diagnosis of Non-Proliferative Breast Disease.

Cytohistological correlation was $88 \%$ while correlation of the Modified Masood score with Histology was $84 \%$. The correlation was statistically significant with a correlation coefficient of 0.832 for CytologyHistology and 0.821 for Modified Masood-Histology. Both the correlation coefficients were significant at the 0.01 level (1-tailed).

Sensitivity of the scoring system was found to be $80 \%$, Specificity $100 \%$ and Positive Predictive Value was $100 \%$, Negative Predictive Value was $92 \%$ for a diagnosis of Carcinoma.

Sensitivity of FNAC was $83 \%$, Specificity was $100 \%$, Positive Predictive Value was $100 \%$ and Negative Predictive value was $93 \%$ for carcinoma.

\section{Discussion}

The study was initiated to evaluate the applicability of Modified Masood Scoring in Cytological Diagnosis of Palpable Breast masses and to compare the scoring system with Cytomorphological Diagnosis. The Histopathological Diagnosis was considered to be the Gold Standard.

According to Barrows, the FNAC positivity for Breast Cancer varies between 48 to $88 \%$. To increase the diagnostic yield of the FNAC, Modified Masood scoring of the aspirates were done. The study helps to categorise the lesion so that aspiration of minimally suspicious lesions is helpful in initiating excisional biopsy.

Modified Masood's scoring gives additional information by eliminating benign cases and improves the diagnostic yield. Application of scoring in a step-wise manner in atypical aspirates can help in selection of cases suitable for biopsy.

The risk of developing subsequent invasive breast cancer is stratified according to the degree of epithelial proliferation and atypia. The risk is 1-fold in women with Non-proliferative Breast disease, 1.9 fold in women with proliferative breast disease and 5.3 in women with carcinoma insitu.

Histological criteria that allow the distinction of these various breast lesions are established. Sneige and Staerkel introduced the concept of using architectural features cytologically and concluded that the application of both cytological and architectural criteria is more reliable than cytology alone in separating proliferative breast lesions.

Dawson et al showed that applying both architectural and cytological criteria enhanced diagnostic accuracy. Cytologically, the architectural features of proliferative breast lesions may be apparent in the larger breast fragments and recapitulate the histologic appearance of these lesions. Slit like lumens, swirling and streaming are noted in proliferative breast lesions without atypia. 


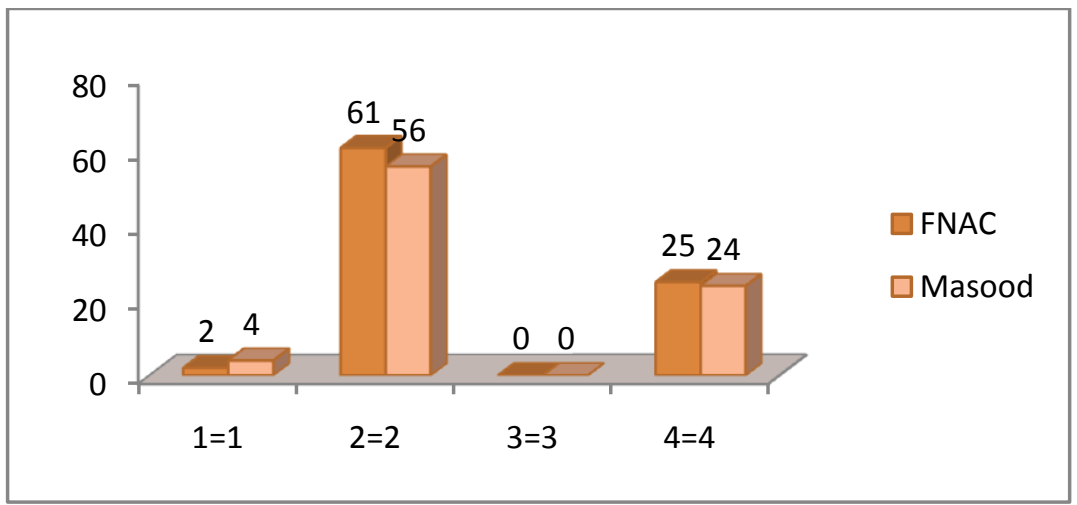

Figure 1 :Concordance of FNAC and Masood scores with Biopsy scores

\begin{tabular}{|c|c|c|c|c|c|}
\hline & & & FNA_Sc & Masood & $\begin{array}{c}\text { B_Scor } \\
\text { e }\end{array}$ \\
\hline \multirow[t]{9}{*}{ Kendall's tau_b } & \multirow[t]{3}{*}{ FNA_Sc } & Correlation Coefficient & 1.000 & $.776^{* *}$ & $.832^{* *}$ \\
\hline & & Sig. (1-tailed) & . & .000 & .000 \\
\hline & & $\mathrm{N}$ & 100 & 100 & 100 \\
\hline & \multirow[t]{3}{*}{ Masood } & Correlation Coefficient & $.776^{* *}$ & 1.000 & $.821^{* *}$ \\
\hline & & Sig. (1-tailed) & .000 & . & .000 \\
\hline & & $\mathrm{N}$ & 100 & 100 & 100 \\
\hline & \multirow[t]{3}{*}{ B_Score } & Correlation Coefficient & $.832^{* *}$ & $.821^{* *}$ & 1.000 \\
\hline & & Sig. (1-tailed) & .000 & .000 & . \\
\hline & & $\mathrm{N}$ & 100 & 100 & 100 \\
\hline \multirow[t]{9}{*}{ Spearman's rho } & \multirow[t]{3}{*}{ FNA_Sc } & Correlation Coefficient & 1.000 & $.829^{* *}$ & $.861^{* *}$ \\
\hline & & Sig. (1-tailed) & . & .000 & .000 \\
\hline & & $\mathrm{N}$ & 100 & 100 & 100 \\
\hline & \multirow[t]{3}{*}{ Masood } & Correlation Coefficient & $.829^{* *}$ & 1.000 & $.856^{* *}$ \\
\hline & & Sig. (1-tailed) & .000 & . & .000 \\
\hline & & $\mathrm{N}$ & 100 & 100 & 100 \\
\hline & \multirow[t]{3}{*}{ B_Score } & Correlation Coefficient & $.861^{* * *}$ & $.856^{* *}$ & 1.000 \\
\hline & & Sig. (1-tailed) & .000 & .000 &. \\
\hline & & $\mathrm{N}$ & 100 & 100 & 100 \\
\hline
\end{tabular}

Figure 2 : Non parametric correlation coefficients

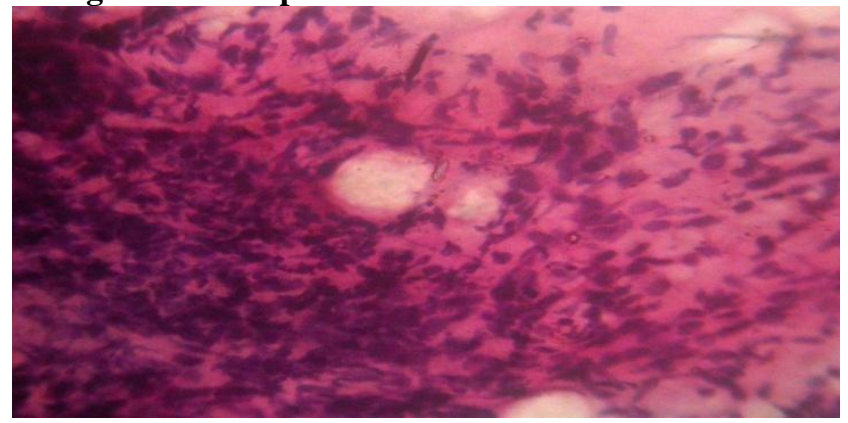

Figure 3: Sclerosing adenosis with a score of 13

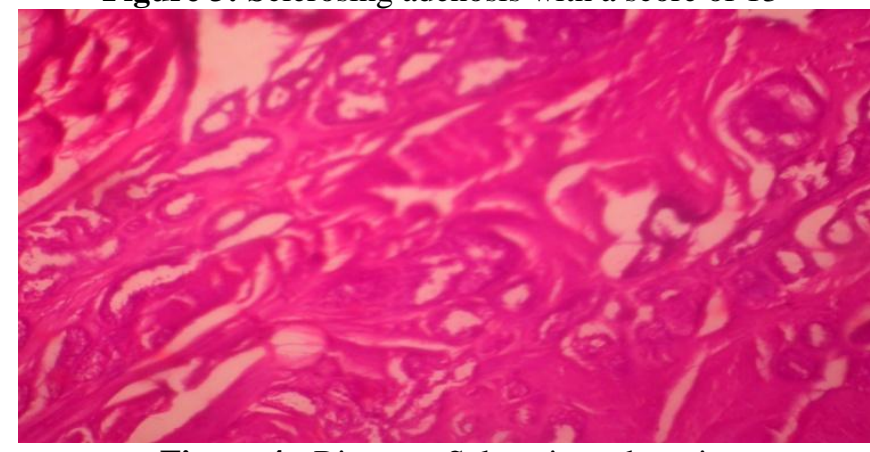

Figure 4 : Biopsy - Sclerosing adenosis 


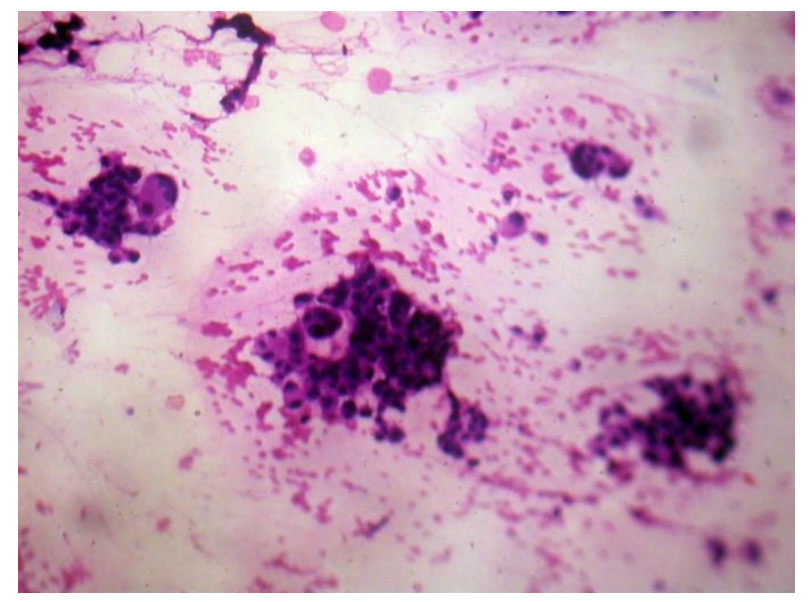

Figure 5 : Intraductal carcinoma with a score of 20

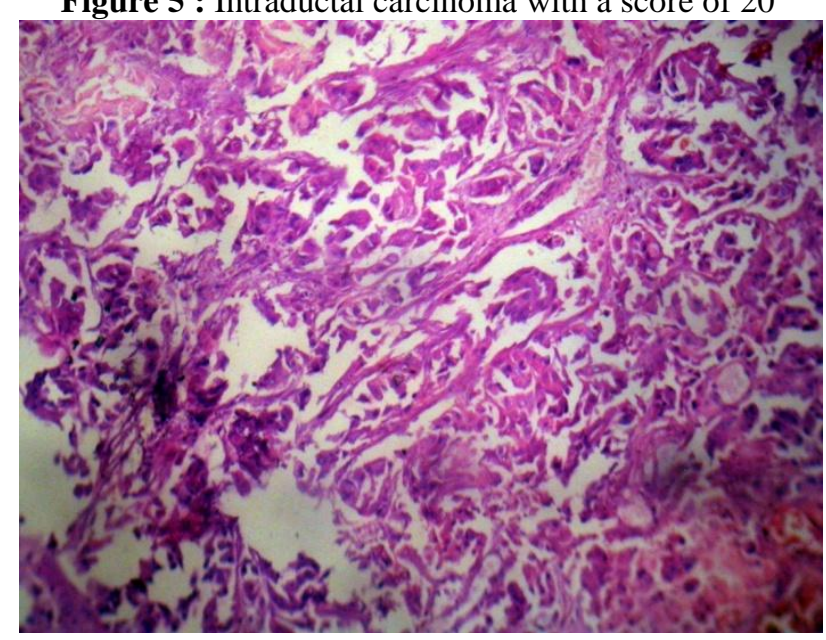

Figure 6 : Biopsy - Intraductal Carcinoma

Rigid sublumina and a micropapillary architecture are features of DCIS. Thomas et al demonstrated that experience and fine tuning of cytological criteria increased the concordance with the histological findings.

All these studies emphasise the importance of adequate sampling to minimise, in particular, underdiagnosis.

Criteria for the cytological diagnosis of Fibroadenoma and Carcinoma are well established. The sensitivity and specificity of Fine Needle Aspiration Cytology for the diagnosis of Fibroadenoma is $86.9 \%$ and 93.8\% respectively, while for Carcinomas, Sensitivity is $89-98 \%$ and Specificity $93-98 \%$.

Proliferative Breast lesions on cytology are categorised into Proliferative Breast Disease without atypia, and Proliferative breast disease with Atypia because it not possible to delineate all the histological entities on FNAC. However, the diagnostic accuracy in this distinction is still unclear, and the cytological features of proliferative breast disease are not well established.

The peak age of incidence of nonproliferative breast disease was found to be in third decade. The peak age of incidence of proliferative breast disease was found to be in third decade and carcinomas peaked at the $6^{\text {th }}$ decades The scoring system is useful in aspirates with cytological diagnosis of proliferative breast disease with atypia. Use of the scoring system can reduce the number of atypical reports and hence limit unnecessary procedures performed on patients.

\section{Conclusion}

100 cases during a period of two years of palpable breast lesions were studied. The cytomorphological analysis by FNAC and Modified Masood system were taken and correlated with histopathological diagnosis. Out of the 100 cases, 4 cases were diagnosed as Non proliferative breast Disease and 66 cases were diagnosed as Proliferative breast disease without atypia. There were no cases under proliferative breast disease with atypia. 30 cases were diagnosed as carcinoma by histopathology

With Modified Masood's scoring, 12 cases were categorized as Nonproliferative breast disease, 59 cases were categorized as proliferative breast disease without atypia, 5 cases were categorized as proliferative 
breast disease with atypia, and 24 cases were categorized as Carcinoma. Sensitivity of the scoring system was found to be $80 \%$, Specificity $100 \%$ and Positive Predictive Value was $100 \%$, Negative Predictive Value was $92 \%$ for a diagnosis of Carcinoma.Hence modified masood`s scoring system correlates well with histopathology and can be used in a stepwise manner to improve the diagnosis of all palpable lesions of breast especially the atypical proliferative lesions of breast and carcinomas .

\section{References}

[1]. Silverberg's Principles and Practice of Surgical Pathology and Cytopathology - 4th edition

[2]. Masood S, Frykberg ER, McLellan GL, Scalapino MC, Mitchum DG, Bullard JB. Prospective evaluation of radiologically directed fine needle aspiration biopsy of nonpalpable breast lesions. Cancer 1990;66:1480-7

[3]. Masood S, Frykberg ER, McLellan GL, Dee S, Mitchum DG, Bullard JB. Cytologic differentiation between proliferative and nonproliferative breast disease in mammographically guided fine needle aspirates. Diagn Cytopathol 1991;7:581-90

[4]. Fine Needle Aspiration of Breast Cancer - Relationship of Clinical Factors to Cytology results in 689 Primary Malignancies. Barrows, et al. Cancer 58:1493-1498, 1986

[5]. Sneige N, Staerkel GA: Fine-needle aspiration cytology of ductal hyperplasia with and without atypia and ductal carcinoma in situ. Hum Pathol 25:485-92, 1994.

[6]. Dawson AE, Mulford DK, Sheils LA. The cytopathology of proliferative breast disease; comparision with features of ductal carcinoma in situ. Am J Clin Pathol 1995;103:438-442.

[7]. Thomas PA, Cangiarella J, Raab SS, Waisman J. Fine needle aspiration biopsy of proliferative breast tissue. Mod Pathol 1995; 8:130-136 